\title{
On Gautschi's conjecture on subrange Jacobi polynomials
}

Research Article

\author{
Gradimir V. Milovanović ${ }^{1,2 *}$ \\ 1 Serbian Academy of Sciences and Arts, Beograd, Mathematical Institute, Belgrade, Kneza Mihaila 35, \\ 11000 Belgrade, Serbia \\ 2 University of Niš, Serbia, Faculty of Sciences and Mathematics, Višegradska 33, 18000 Niš, Serbia
}

\begin{abstract}
In this short note, we give a partial positive answer to Gautschi's conjecture about the monotonicity of positive zeros of subrange Jacobi polynomials, stated recently in his paper [Numer. Algorithms 79 (2018), no. 3, 759-768].

MSC: $\quad 33 \mathrm{C} 47,65 \mathrm{D} 30$

Keywords: Orthogonal polynomial • Subrange Jacobi polynomials • Zeros • Monotonicity
\end{abstract}

\section{Introduction}

Recently Walter Gautschi [6] has considered zeros of (monic) subrange Jacobi polynomials of degree $n$, in notation $\pi_{n}(\cdot)=\pi_{n}^{(\alpha, \beta)}(\cdot ; c)$, which are orthogonal on $[-c, c], 0<c<1$, with respect to the weight function $w(x)=$ $(1-x)^{\alpha}(1+x)^{\beta}, \alpha, \beta>-1$. Such kind of orthogonal polynomials on a strict subinterval of $[-1,1]$, including their numerical computation, as well as the related Gaussian quadrature rules, were introduced and studied also by Gautschi in his earlier paper [5]. It is interesting that in [2] and [3] Da Fies and Vianello, in connection with subperiodic trigonometric quadrature, used sub-range Chebyshev polynomials orthogonal with respect to the Chebyshev weight of the first kind $(\alpha=\beta=-1 / 2)$ on the interval $[-c, c]$, where $c=\sin (\omega / 2)$, with $0<\omega<\pi$, in order to construct some kind of Gaussian product formulas for integration over circular and annular sectors, circular zones, etc.

As in [7], in his study of the monotonicity behavior of the zeros $x_{\nu}$ of the orthogonal polynomial $\pi_{n}^{(\alpha, \beta)}(x ; c)$, Gautschi started from the respective Gaussian quadrature formula,

$$
\int_{-c}^{c} p(x) w(x) \mathrm{d} x=\sum_{\nu=1}^{n} A_{\nu}(c) p\left(x_{\nu}(c)\right), \quad p \in \mathcal{P}_{2 n-1}
$$

* E-mail: gvm@mi.sanu.ac.rs 
where $x_{\nu}=x_{\nu}(c)$ are the zeros of the $n$ th-degree subrange Jacobi polynomial $\pi_{n}^{(\alpha, \beta)}(x ; c)$ and $A_{\nu}(c)$ are the corresponding weight coefficients (Christoffel numbers). Then, differentiating (1) with respect to $c$,

$$
p(c) w(c)+p(-c) w(-c)=\sum_{\nu=1}^{n} \frac{\mathrm{d} A_{\nu}(c)}{\mathrm{d} c} p\left(x_{\nu}(c)\right)+\sum_{\nu=1}^{n} A_{\nu}(c) p^{\prime}\left(x_{\nu}(c)\right) \frac{\mathrm{d} x_{\nu}}{\mathrm{d} c}
$$

using A. Markov idea (cf. [8, §6.12]), and putting $p(x)=\left[\pi_{n}(x)\right]^{2} /\left(x-x_{\nu}\right)\left(p \in \mathcal{P}_{2 n-1}\right.$, because $\left.\pi_{n}\left(x_{\nu}\right)=0\right)$, he concluded that

$$
w(c) \pi_{n}(c)^{2}\left\{\frac{1}{c-x_{\nu}}-\left[\frac{\pi_{n}(-c)}{\pi_{n}(c)}\right]^{2} \frac{w(-c)}{w(c)} \cdot \frac{1}{c+x_{\nu}}\right\}=A_{\nu}(c)\left[\pi_{n}^{\prime}\left(x_{\nu}\right)\right]^{2} \frac{\mathrm{d} x_{\nu}}{\mathrm{d} c}
$$

because for all $\nu, p\left(x_{\nu}\right)=0$ and $p^{\prime}\left(x_{\nu}\right)=\left[\pi_{n}^{\prime}\left(x_{\nu}\right)\right]^{2}$.

In the symmetric ultraspherical case $(\alpha=\beta)$, when $w(-c)=w(c)$ and $\pi_{n}(-c)^{2}=\pi_{n}(c)^{2},(2)$ reduces to

$$
w(c) \pi_{n}(c)^{2} \frac{2 x_{\nu}}{c^{2}-x_{\nu}^{2}}=A_{\nu}(c)\left[\pi_{n}^{\prime}\left(x_{\nu}\right)\right]^{2} \frac{\mathrm{d} x_{\nu}}{\mathrm{d} c},
$$

wherefrom for $x_{\nu}>0$, Gautschi concluded that $\mathrm{d} x_{\nu} / \mathrm{d} c>0$ and, in this way, he proved that all positive zeros $x_{\nu}$ of the subrange ultraspherical polynomials $(\alpha=\beta)$, orthogonal on $[-c, c], 0<c<1$, are monotonically increasing as functions of $c$ (see [6, Theorem 1]).

In the general case when $-1<\alpha<\beta$, for any $n \in \mathbb{N}$ and any $c$ with $0<c<1$, because of $\pi_{n}^{(\alpha, \beta)}(x ; c)=$ $\pi_{n}^{(\beta, \alpha)}(-x ; c)$ and

$$
\frac{w(-c)}{w(c)}=\left(\frac{1-c}{1+c}\right)^{\beta-\alpha}
$$

(2) reduces to

$$
w(c) \pi_{n}(c)^{2}\left\{\frac{1}{c-x_{\nu}}-\left[\frac{\pi_{n}(-c)}{\pi_{n}(c)}\right]^{2}\left(\frac{1-c}{1+c}\right)^{\beta-\alpha} \frac{1}{c+x_{\nu}}\right\}=\lambda_{\nu}(c)\left[\pi_{n}^{\prime}\left(x_{\nu}\right)\right]^{2} \frac{\mathrm{d} x_{\nu}}{\mathrm{d} c}
$$

and Gautschi has stated the following wondrous conjecture:

Conjecture 1.1 .

For any $n \geq 1, \alpha>-1, \beta>-1$ with $\alpha<\beta$, and for any $c$ with $0<c \leq 1$, there holds

$$
\left[\frac{\pi_{n}(-c)}{\pi_{n}(c)}\right]^{2}\left(\frac{1-c}{1+c}\right)^{\beta-\alpha}<1
$$

where $\pi_{n}(\cdot)=\pi_{n}^{(\alpha, \beta)}(\cdot ; c)$ is the subrange Jacobi polynomial of degree $n$ orthogonal on $[-c, c]$ with respect to the weight function $w(x)=(1-x)^{\alpha}(1+x)^{\beta}$.

Empirical evidence in support of the conjecture is provided in [6, Appendix B].

In this note, for a given $c \in(0,1)$ we give proof of this conjecture in the domain

$$
A(c)=\left\{(\alpha, \beta) \mid \beta \geq \frac{1-c}{1+c} \alpha \text { if }-1<\alpha \leq 0\right\} \bigcup\left\{(\alpha, \beta) \mid \beta \geq \frac{1+c}{1-c} \alpha \text { if } \alpha \geq 0\right\}
$$


The problem is still open in the domains

$$
B(c)=\left\{(\alpha, \beta) \mid \alpha \leq \beta<\frac{1-c}{1+c} \alpha \text { if }-1<\alpha<0\right\}
$$

and

$$
C(c)=\left\{(\alpha, \beta) \mid \alpha<\beta<\frac{1+c}{1-c} \alpha \text { if } \alpha \geq 0\right\} .
$$

\section{Proof of Conjecture (1.1) in the domain $\boldsymbol{A}(\boldsymbol{c})$}

In this section we consider the problem transformed from $[-c, c]$ to $[-1,1]$ by the simple change of variables $x=c t$. The equivalent form of Conjecture (1.1) can be formulated for the weight function on $[-1,1]$, given by

$$
W(t)=w(c t)=(1-c t)^{\alpha}(1+c t)^{\beta}, \quad 0<c<1,-1<\alpha<\beta,
$$

and the corresponding monic orthogonal polynomials $\Pi_{n}(t)$, given by $\Pi_{n}(t)=\pi_{n}(c t) / c^{n}, n \in \mathbb{N}$.

Then the inequality (3) in Conjecture 1.1 becomes

$$
\left(\frac{\Pi_{n}(-1)}{\Pi_{n}(1)}\right)^{2} \frac{W(-1)}{W(1)}<1
$$

i.e.,

$$
D=W(1) \Pi_{n}(1)^{2}-W(-1) \Pi_{n}(-1)^{2}>0 .
$$

Since

$$
\frac{\mathrm{d}}{\mathrm{d} t}\left(W(t) \Pi_{n}(t)^{2}\right)=W^{\prime}(t) \Pi_{n}(t)^{2}+2 W(t) \Pi_{n}(t) \Pi_{n}^{\prime}(t),
$$

we have

$$
\begin{aligned}
D & =\int_{-1}^{1}\left[W^{\prime}(t) \Pi_{n}(t)^{2}+2 W(t) \Pi_{n}(t) \Pi_{n}^{\prime}(t)\right] \mathrm{d} t \\
& =\int_{-1}^{1} W^{\prime}(t) \Pi_{n}(t)^{2} \mathrm{~d} t+2 \int_{-1}^{1} W(t) \Pi_{n}(t) \Pi_{n}^{\prime}(t) \mathrm{d} t
\end{aligned}
$$

i.e.,

$$
D=\int_{-1}^{1} W^{\prime}(t) \Pi_{n}(t)^{2} \mathrm{~d} t
$$

because of the orthogonality, the second integral in (8) is equal to zero.

Since

$$
W^{\prime}(t)=-\alpha c(1-c t)^{\alpha-1}(1+c t)^{\beta}+\beta c(1-c t)^{\alpha}(1+c t)^{\beta-1},
$$

i.e.,

$$
W^{\prime}(t)=c \frac{W(t)}{1-c^{2} t^{2}} \Phi(c t)
$$


where

$$
\Phi(z)=\beta-\alpha-(\beta+\alpha) z
$$

and $c t \in(-1,1)$, we see that

$$
\Phi(-1)=2 \beta, \quad \Phi(1)=-2 \alpha .
$$

Figure 1. Different domains for $(\alpha, \beta) \in \mathbb{R}^{2}$

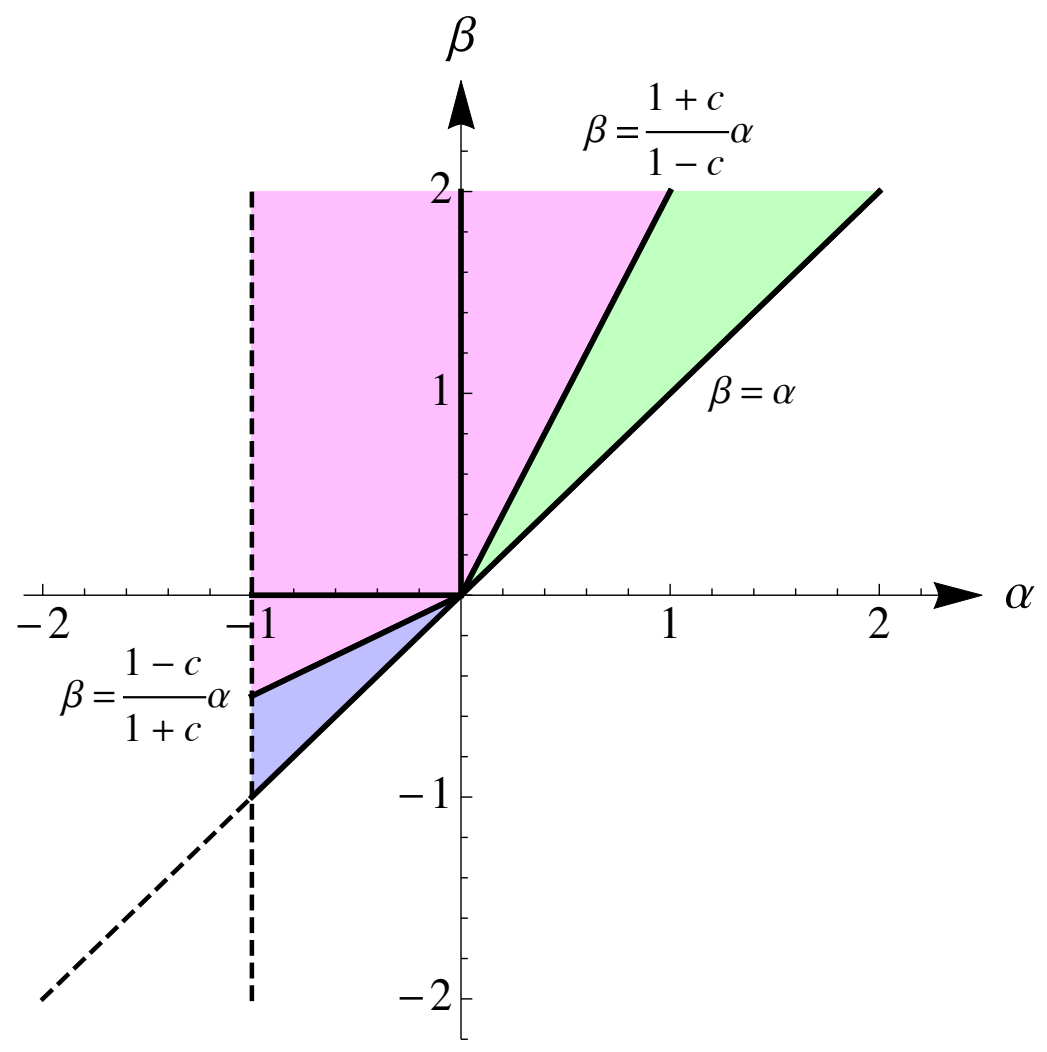

Evidently, $\Phi(c t) \geq 0$ for each $c \in(0,1)$ and $t \in(-1,1)$, if $\beta \geq 0$ and $\alpha \leq 0$, i.e., if

$$
(\alpha, \beta) \in\{(\alpha, \beta) \mid-1<\alpha \leq 0, \beta \geq 0\}
$$

(see Figure 1, colored part in the second quadrant).

However, the conjecture is true if

$$
\beta-\alpha-|\beta+\alpha| c \geq 0
$$

that is,

$$
c \leq \frac{\beta-\alpha}{|\beta+\alpha|}
$$


(as always, $\beta-\alpha>0$ ). If the right-hand side is $\geq 1$, the conjecture is true unrestrictedly, for all $0<c<1$. This is the case, as we mentioned before, if $\beta \geq 0$ and $\alpha \leq 0$ (colored part in the second quadrant).

In the square $-1<\alpha<0,-1<\beta<0$, the conjecture is true if

$$
c \leq \frac{|\alpha|-|\beta|}{|\alpha|+|\beta|}=\frac{\alpha-\beta}{\alpha+\beta},
$$

i.e., when $\beta>\alpha(1-c) /(1+c)$.

In the domain $\alpha>0, \beta>0, \beta>\alpha,(7)$ holds if

$$
c \leq \frac{\beta-\alpha}{\beta+\alpha}
$$

i.e., when $\beta>\alpha(1+c) /(1-c)$.

That summarizes the current state of the conjecture.

\section{Theorem 2.1.}

For each $c$ with $0<c \leq 1$, the inequality (7), i.e., (3), holds if $(\alpha, \beta) \in A(c)$, where $A(c)$ is a domain in $\mathbb{R}^{2}$ defined by (4).

Thus, the conjecture is true in the domain in $\mathbb{R}^{2}$, which is colored magenta in Figure 1.

The problem is still open in the domains $B(c)$ and $C(c)$, given by (5) and (6), respectively. These domains are colored in blue and green in Figure 1.

\section{Remark 2.1.}

For orthogonal polynomials and Gaussian quadratures see books [4] and [9]. For numerical and symbolic constructions of orthogonal polynomials and Gaussian quadrature formulas there is a software package in MATHEMATICA (see [1] and [10]).

\section{Acknowledgements}

This work was supported in part by the Serbian Academy of Sciences and Arts ( $\Phi-96)$.

\section{References}

[1] A.S. Cvetković, G.V. Milovanović, The Mathematica Package "OrthogonalPolynomials", Facta Univ. Ser. Math. Inform. 9, (2004), 17-36. MR2122752

[2] G. Da Fies, M. Vianello, Trigonometric Gaussian quadrature on subintervals of the period, Electron. Trans. Numer. Anal. 39 (2012), 102-112. MR2995775

[3] G. Da Fies, M. Vianello, Algebraic cubature on planar lenses and bubbles, Dolomites Res. Notes Approx. 5 (2012), $7-12$. 
[4] W. Gautschi, Orthogonal polynomials: computation and approximation. Numerical Mathematics and Scientific Computation, Oxford University Press, New York, 2004. MR2061539

[5] W. Gautschi, Sub-range Jacobi polynomials, Numer. Algoritms 61 (2012), no. 4, 649-657, DOI 10.1007/s11075-012-9556-z. MR2995225

[6] W. Gautschi, On the zeros of subrange Jacobi polynomials, Numer. Algoritms 79 (2018), no. 3, 759-768. DOI 10.1007/s11075-017-0459-x. MR3869504

[7] W. Gautschi, G. V. Milovanović, Binet-type polynomials and their zeros, Electron. Trans. Numer. Anal. 50 (2018), 52-70. DOI: 10.1553/etna_vol50s52. MR3882260

[8] Gabor Szegö, Orthogonal polynomials, 4th edn., Colloquium Publications, vol. 23. American Mathematical Society, Providence, R.I., 1975. MR0372517

[9] G. Mastroianni, G. V. Milovanović, Interpolation processes. Basic theory and applications, Springer Monographs in Mathematics, Springer-Verlag, Berlin, 2008. MR2457729. DOI 10.1007/978-3-540-68349-0

[10] G.V. Milovanović, A.S. Cvetković, Special classes of orthogonal polynomials and corresponding quadratures of Gaussian type, Math. Balkanica, 26 (2012), 169-184. MR2977032 